\title{
3D geometric morphometric analysis of variation in the human lumbar spine
}

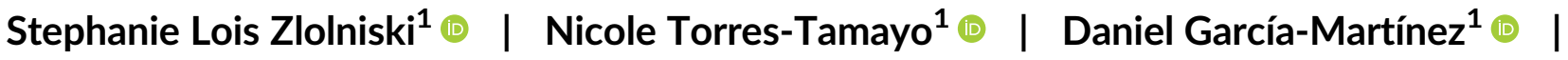 \\ Esther Blanco-Pérez $^{2}$ | Federico Mata-Escolano ${ }^{3}$ | Alon Barash ${ }^{4}$ | Shahed Nalla ${ }^{5,6}$ | \\ Sandra Martelli $^{7}$ | Juan A. Sanchis-Gimeno ${ }^{8}$ | Markus Bastir ${ }^{1}$ (6)
}

${ }^{1}$ Paleoanthropology Group, Museo Nacional de Ciencias Naturales (CSIC), Madrid, Spain

${ }^{2}$ Department of Radiology, Hospital de La Ribera, Valencia, Spain

${ }^{3} \mathrm{CT}$ and MRI Unit, ERESA, Department of Radiology, General University Hospital, Valencia, Spain

${ }^{4}$ Faculty of Medicine in the Galilee, Bar Ilan University, Ramat Gan, Israel

${ }^{5}$ Department of Human Anatomy and Physiology, Faculty of Health Sciences, University of Johannesburg, Johannesburg, South Africa

${ }^{6}$ Evolutionary Studies Institute and Centre for Excellence in PalaeoSciences, University of the Witwatersrand, Johannesburg, South Africa

${ }^{7}$ Centre for Integrative Anatomy (CIA), Department of Cell and Developmental Biology, Faculty of Life Sciences, University College London, London, UK

${ }^{8}$ Giaval Research Group, Department of Anatomy and Human Embryology, Faculty of Medicine, University of Valencia, Valencia, Spain

\section{Correspondence}

Stephanie Lois Zlolniski and Markus Bastir, Paleoanthropology Group, Museo Nacional de Ciencias Naturales (CSIC), Madrid, Spain. Email: stephanie.lois.zlolniski@gmail.com (S. L. Z.) and mbastir@mncn.csic.es (M. B.)

\section{Funding information}

Ministry of Economy, Industry and Competitiveness, Grant/Award Number: CGL-2015-63648-P; ERESA Clinic Research Foundation, Grant/Award Number: BF14_005

\begin{abstract}
Objectives: The shape of the human lumbar spine is considered to be a consequence of erect posture. In addition, several other factors such as sexual dimorphism and variation in genetic backgrounds also influence lumbar vertebral morphology. Here we use 3D geometric morphometrics (GM) to analyze the 3D morphology of the lumbar spine in different human populations, exploring those potential causes of variation.

Material and methods: We collected 390 (semi) landmarks from 3D models of the CT scans of lumbar spines of seven males and nine females from a Mediterranean population (Spain, Israel) and seven males and either females from a South African population for geometric morphometric (GM) analysis. We carried out Generalized Procrustes Analysis, Principal Components, and Regression analyses to evaluate shape variation; and complemented these analyses with the Cobb Method.

Results: The Mediterranean sample was considerably more lordotic than the South African sample. In both populations, female lumbar spines showed proportionally narrower and more craniocaudally elongated lumbar segments than in males. In addition, the point of maximum curvature in females tended to be located more inferiorly than in males.

Discussion: Our results show that sexual dimorphism is an important factor of lumbar spine variation that mainly affects features of lumbar spine robustness (height proportions) and the structure-but not the degree-of its curvature. Differences in lordosis, however, are clearer at the inter-population level. This reflects previous conflicting studies casting doubts on pregnancy as an adaptive factor influencing lordosis. Other factors, for example, shape of the individual lumbar vertebrae and intervertebral discs and their relative proportions within the lumbar spine should be considered when exploring variation in vertebral column morphology.
\end{abstract}

\section{KEYWORDS}

lordosis, Mediterranean Caucasian, population variation, sexual dimorphism, South African 


\section{1 | INTRODUCTION}

A permanent lumbar lordosis is one of the key features of the hominin vertebral column (Kummer, 1975; Lovejoy, 2005). Its presence is a good indicator of habitual bipedalism. The ventrally convex lumbar spine curvature in the mid-sagittal plane helps the pelvis stabilize and distribute the body weight over the lower limbs, as it assists in positioning the trunk's center of mass above the hips and brings the line of gravity close to the acetabula (Farfan, 1995; Kummer, 1975).

The shape of the vertebral bodies and particularly the intervertebral discs results from the development of the lumbar lordosis during postnatal ontogeny: a greater dorsal wedging (an element's dorsal height smaller than its ventral height) and a greater inclination on the horizontal plane of the vertebral segments (vertebral body and the immediate disc below) will lead to a greater lordosis angle (Been, Barash, \& Marom, 2010; Shefi, Soudack, Konen, \& Been, 2013).

Furthermore, the degree of lumbar lordosis varies greatly throughout an individual's life history-during postnatal ontogeny, the lumbar lordosis is established and reaches its initial adult configuration shortly after puberty (Reichmann \& Lewin, 1971; Shefi et al., 2013). During adulthood, the lumbar lordosis can change further with the senescence process (Dreischarf et al., 2014; Jentzsch, Geiger, König, \& Werner, 2017; Korovessis, Stamatakis, \& Baikousis, 1998; Pries, Dreischarf, Bashkuev, Putzier, \& Schmidt, 2015). These changes are closely related to factors such as bone and soft tissue degeneration (Bogduk, 2005), genetic make-up variability in different populations (i.e., inter-population variation) and sexual dimorphism. This study will focus on the latter two factors.

Concerning the inter-population variation, other than the earliest anthropological studies (e.g., Turner, 1886), there have not been many studies focusing mainly on this type of variation. It is usually more common for a lumbar morphology study to focus on a particular parameter such as the lumbosacral angle or the sacral slope but with little consideration for differences in population selection. For example, Okpala (2014) in a more recent study focused on the sacral slope in a Nigerian population resulting in a greater lumbar curvature between the measured sample and reference populations studied by similar methods by other authors (e.g., Hellems \& Keats, 1971; Maduforo et al., 2012). The differences were not very large but nonetheless significant. Likewise, Patrick (1976) analyzed differences in lumbar spine morphology within another Nigerian population reaching similar conclusions while applying a flexicurve ruler (described by Milne and Lauder (1974)) from the seventh cervical vertebra to the lumbosacral joint. The choice of population is rarely explained in these studies, other than implying relatively easy access to local data. This leads to an interesting line of questioning in that inter-population variation is generally poorly quantified or not addressed at all.

Some of the few recent inter-population studies are by Hanson, Magnusson, and Simonsen (1998) and by Merrill et al. (2018) who analyzed two and three populations of varying genetic backgrounds, respectively (African-American vs. continental European; and European-American vs. African-American and vs. Hispanic). Hanson et al. (1998) reported differences in lumbar spine morphology, that is, less lumbar lordosis in African Americans than in the continental Europeans using a specially designed device to measure the sacral angle and the lumbosacral curve on dissected spines. Merrill et al. (2018) by measuring lateral lumbosacral $X$-rays found a larger lumbar lordosis in the Hispanic population than in the European-American, and no other population differences regarding lordotic curvature. Nevertheless, these studies are not very detailed on what inter-population variation (i.e., genetics) is based on. Patrick (1976) undertook a comparison of the Nigerian population he studied with already published data from European populations and found statistically significant differences between these populations, with Nigerians on average having a larger lumbosacral angle than the Europeans. He considered either inter-population variation in genetic make-up or local customs of carrying heavy loads as possible explanations for this. On the other hand, Mosner, Bryan, Stull, and Shippee (1989) found differences between the apparent lordosis (gluteal prominence) of African-American and European-American females, but not between the actual lordosismeasured on standing lateral radiographs-of both populations.

Looking at sexual dimorphism, previous research using the Cobb method (Cobb, 1948) on lateral X-rays to quantify the degree of lumbar lordosis, has shown sexual variability within both vertebral morphology and the curvature of the lumbar spine (Vialle et al., 2005). Female vertebral segments are relatively taller and narrower (see our designation for measurements in Figure 1): Taylor and Twomey (1984) found a greater height/transverse diameter index in the female lumbar vertebrae of their population, which was composed both of children (difference of 0.04) and adults (difference of 0.09). Additionally, adult female elements show greater dorsal wedging than males. This, in turn, results in a more lordotic female lumbar spine (Poussa et al., 2005; Vialle et al., 2005; Whitcome, Shapiro, \& Lieberman, 2007). Vialle et al. (2005) after digitally measuring lateral radiographs, found a difference of $4.8^{\circ}$ in the lumbar curvature between adult females and males. Poussa et al. (2005) by performing spinal pantographs (Willner, 1981), also reported a female lordotic curvature which was $1.58^{\circ}$ greater than in males in their cohort of children and young adults aged between 11 and 22 years. Some studies interpreted this greater female lordotic curvature as an adaptation to

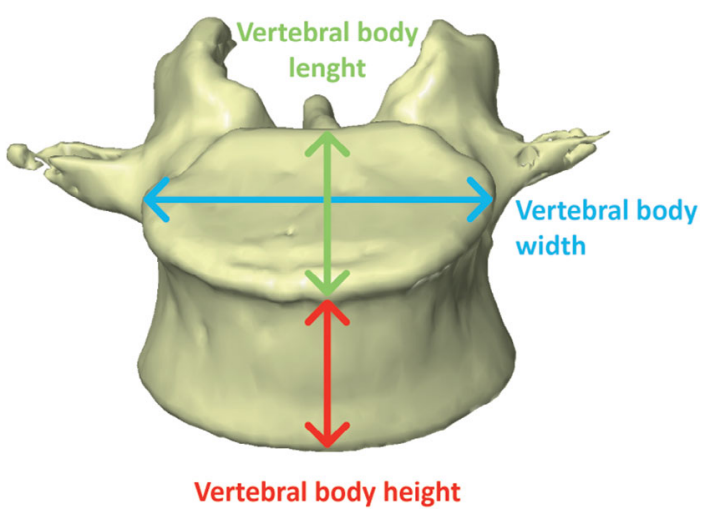

FIGURE 1 Vertebral body measurements used in this work 
pregnancy, arguing that a greater lordosis could improve the body equilibrium of pregnant females following static principles (Bailey, Sparrey, Been, \& Kramer, 2016; Masharawi et al., 2010; Whitcome et al., 2007).

Nevertheless, there is a number of studies investigating different adult populations and applying different methodologies, which did not find sexual dimorphism neither in lordosis (Hasegawa et al., 2017; Jentzsch et al., 2017; Kalichman, Li, Hunter, \& Been, 2011) nor in the wedging of the vertebral segments (Fazzalari, Manthey, \& Parkinson, 2001). Legaye, Duval-Beaupère, Hecquet, and Marty (1998) even found a greater mean lordotic angle in males than in females. However, all these studies did not take inter-population variability into consideration.

One of the possible reasons-among other factors described later-why some studies report sexual dimorphism and others do not might be explained by the different mean curvatures found in each research, which could arise from the different type of data and number of vertebral elements used by each author when assessing the variation in the lumbar spine. Lordosis and wedging angles are usually measured by the Cobb method (Cobb, 1948), which has been widely used due to its easy application and reproducibility (Vrtovec, Pernus, \& Likar, 2009). When applying this method, some authors prefer to measure the lordotic curvature from the superior endplate of L1 to the inferior endplate of L5 (Vialle et al., 2005), while others also include the sacral endplate, thus including the effect of the L5-S1 intervertebral disc (Shefi et al., 2013). These angles, as well as other measurements such as vertebral heights and lengths, are usually taken directly on lateral X-rays (Taylor \& Twomey, 1984; Vialle et al., 2005). Furthermore, study populations vary in different aspects such as sample's size, posture, body mass index, mean age and age range, presence of lumbosacral transitional vertebrae, genetic differences, and so forth. These characteristics can have an influence on the degree of sexual dimorphism of the lumbar lordosis observed (Jentzsch et al., 2017; Korovessis et al., 1998; Pries et al., 2015).

However, as Fazzalari et al. (2001) pointed out, 2D radiographic techniques are not the most suitable to appreciate the threedimensionality of the lumbar vertebral elements. On the one hand, conventional radiographs suffer from image distortion (Bittersohl et al., 2013; Chiron et al., 2017; Tardieu, Hasegawa, \& Haeusler, 2017). On the other hand, both angular and linear measures-taken from said radiographs-do not account for spatial features of lumbar vertebral alignment such as the relation of vertebral bodies and intervertebral discs (Lois Zlolniski et al., 2017), the serial relation of vertebral elements position and height or the immediate location of the point of maximum curvature of the lumbar lordosis. It should be mentioned that Vaz, Roussouly, Berthonnaud, and Dimnet (2002) and Vialle et al. (2005) showed that specific spatial arrangements of vertebral elements could similarly fulfill biomechanical functions despite giving rise to different angles or wedging values. For example, the same pelvic incidence value could be matched with varying kyphotic and lordotic angles in order to find the optimal posture. Following this first idea and in an attempt at including the three-dimensionality of the lumbar spine, Kalichman et al. (2011) and Zhou, McCarthy,
McGregor, Coombs, and Hughes (2000) used 3D CT scans, and Fazzalari et al. (2001), Masharawi et al. (2010) and Reichmann and Lewin (1971) worked directly on dissected spines and vertebrae. In addition, low-dose radiology techniques that allow for 3D reconstruction of 2D images have been developed. In this case, EOS imaging stands out as it combines simultaneous acquisition of lateral and frontal radiographies and allows for a $3 \mathrm{D}$ reconstruction of the spine in the standing position without any distortion (Dubousset et al., 2005; Hasegawa et al., 2017; Le Huec \& Hasegawa, 2016; Tardieu et al., 2017). Such 3D studies have enhanced the possibility to explore combinations of spinal features and how these could interact, for example, during the development of a greater lordotic curvature in females (Masharawi et al., 2010).

This study goes one step further in analyzing shape variation of the $3 \mathrm{D}$ configuration of the lumbar vertebral bodies and its contribution to lumbar spine morphology and curvature. We used 3D geometric morphometrics (O'Higgins, 2000)-which allows for rigorous quantification of size and shape-to explore potential factors of variation in the lumbar spine morphology such as variability due to different genetic backgrounds and sexual dimorphism. In addition, we compare these configurations with the classic standard measurements obtained by the Cobb method (Cobb, 1948), in order to qualify the additional information a 3D geometric morphometrics study might yield.

\section{2 | MATERIAL AND METHODS}

The methodology used in this study is 3D geometric morphometrics (3D-GM), which is defined as the statistical analysis of an object's size and shape in a virtual space (Zelditch, Swiderski, \& Sheets, 2012). This information is obtained from Cartesian landmark and semilandmark coordinates located on specific points on the object (Mitteroecker \& Gunz, 2009). The precise definition of these landmarks and semilandmarks allows for a rigorous quantification of morphological changes and a clear visualization of differences between complex forms, which otherwise would be difficult to distinguish (Bookstein, 1991; Mitteroecker \& Gunz, 2009; O'Higgins, 2000; Zelditch et al., 2012).

\section{1 | Sample}

We reconstructed 3D models of the lumbar spine of 31 subjects in supine position (16 Mediterraneans and 15 South Africans) using their CT scans from three different medical institutions: Mount Carmel Hospital in Haifa (Israel), ERESA Medical Group for Special Radiological Explorations in Valencia (Spain) and Charlotte Maxeke Academic Hospital through the Department of Human Anatomy and Physiology, Faculty of Health Sciences, University of Johannesburg (South Africa). The study was approved by the local Ethics Committee of the University of Valencia (IRB number H1417174744011) and the Human Research Ethics Committee of the Charlotte Maxeke Academic Hospital (Clearance Certificate NO. M130844). There was no examination from an Ethics Committee from Israel, but, as the three samples were anonymized, the study could be conducted in accordance with the 
TAB LE 1 Description of the samples analyzed

\begin{tabular}{|c|c|c|c|c|c|c|c|}
\hline & \multicolumn{3}{|c|}{ Mediterranean sample } & \multicolumn{3}{|c|}{ South African sample } & \multirow[b]{2}{*}{$p$-value } \\
\hline & Mean & Range & $S D$ & Mean & Range & $S D$ & \\
\hline Age (years) & 48.9 & $18-73$ & 18.2 & 42.4 & $19-65$ & 15.4 & .268 \\
\hline Body height (cm) & 169 & $156-183$ & 8.7 & 168.8 & $158-182$ & 8.2 & .945 \\
\hline Body weight (kg) & 63.7 & $50-78$ & 8.1 & 63.2 & $55-76$ & 7.3 & .850 \\
\hline Body mass index $\left(\mathrm{kg} / \mathrm{m}^{2}\right)$ & 22.2 & $20.0-24.6$ & 1.6 & 22.1 & $19.7-23.0$ & 1.0 & .799 \\
\hline
\end{tabular}

Declaration of Helsinki (Goodyear, Krleza-Jeric, \& Lemmens, 2007), as with the study sample used by Torres-Tamayo et al. (2018).

All subjects underwent a CT scan study on the basis of suspected metastasis of either breast, melanoma, or colon neoplasm. In order to be included in our study all the subjects had to be free of metastases and needed to show a free-of-anomalies and nonpathological, common configuration lumbar spine (i.e., five vertebrae, no fractures, no scoliosis, etc.). In addition, if potential study subjects' patient records also included suffering from low back pain or spinal trauma, they were excluded from the study in order to avoid factors that could affect the lumbar curve and thus the outcome of the study.

The sample was divided into four groups defined by sex and population. Consequently, we analyzed a Caucasian sample of seven males and nine females from the circum-Mediterranean area (Spain and Israel; hereafter termed "Mediterraneans") and a sample from South Africa of seven males and eight females (hereafter termed "South Africans"). The reason for this population classification is that we assumed that Spaniards and Israelis share Caucasian skeletal features, which differ from South Africans due to ancestry (Behar et al., 2007; Okpala, 2014; Patrick, 1976).

Table 1 presents the demographic characteristics of the analyzed population samples. No differences were found between Mediterranean and South African samples. Also, no differences in age $(p=.225)$ and body mass index $(p=.885)$ were found between Mediterranean and South African women. The same applies to Mediterranean and South African men ( $p=.785$ for age, and $p=.820$ for body mass index). As a result, we considered the population samples to be a good match for comparison, with Mediterranean or South African origin being the biggest difference between them.

The lumbar spines, consisting of five lumbar vertebral bodies (L1L5) and the base of the sacrum (S1), were segmented using Mimics 8 software to obtain the 3D models (http://www.materialise.com/en/ medical/software/mimics). The 3D models were post-processed-that is, trimmed, cleaned, and smoothed-using Artec Studio software (www.Artec3D.com), which facilitated anatomical measurements in a virtual environment. Finally, the 3D models were imported into Viewbox 4 software (www.dhal.com) for (semi)landmark digitization.

\section{2 | (Semi)landmarks configuration}

We defined and collected 390 landmarks and semilandmarks on each lumbar spine 3D model-33 anatomical landmarks, 177 curve semilandmarks and 180 surface semilandmarks (Figure 2). Landmarks were

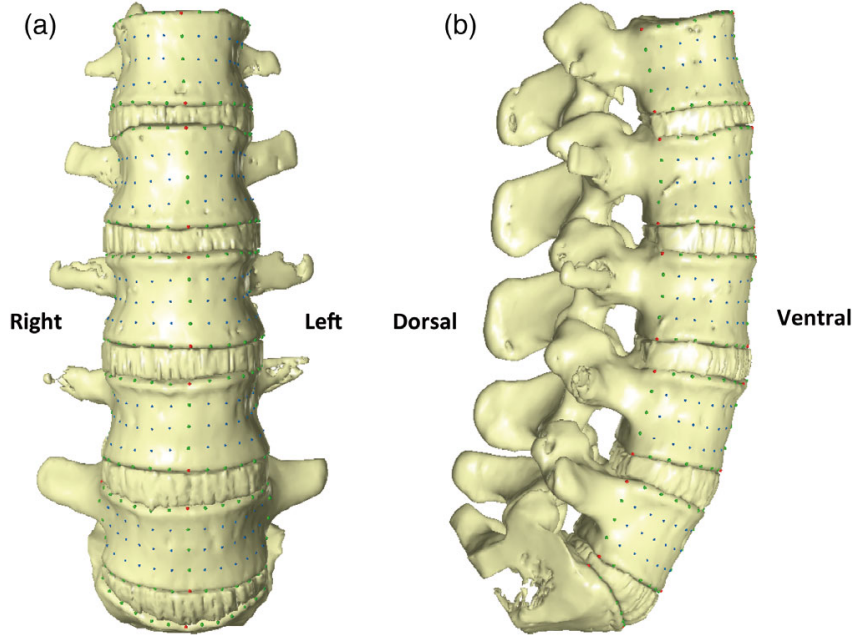

FIGURE 2 3D landmarks location in (a) frontal and (b) left lateral view. Red: 33 landmarks; Green: 177 curve semilandmarks; Blue: 180 surface semilandmarks

placed at: the point of union of the right and left pedicles to the superior margin of the intervertebral surface (on the annular epiphysis) of each vertebral body, on the most lateral parts (right and left) of the base of the sacrum, on the most anterior point of the superior margin of each intervertebral surface and on the inferior margin of the intervertebral surface of each vertebral body, following a straight line from the ones on the superior margin. Curve semilandmarks were distributed in four curves of six semilandmarks along the borders of both intervertebral surfaces on each vertebral body, outlining at the same time the borders of the intervertebral discs. Furthermore, three more curves of three semilandmarks were drawn between the landmarks on the superior intervertebral surface of each vertebra and their corresponding ones on the inferior surface. Lastly, 36 surface semilandmarks were placed on each vertebral body. Thus, this template configuration collected $3 \mathrm{D}$ shape variation related to the vertebral bodies and the associated intervertebral discs.

The semilandmarks underwent a sliding process along tangent lines or tangent planes to their corresponding curves or surfaces, respectively (Gunz \& Mitteroecker, 2013; Gunz, Mitteroecker, \& Bookstein, 2005). This process removes the effect of arbitrary position until the optimal spacing is achieved. In other words, once resliding is applied, there is a minimal morphometric difference (bending energy) between each specimen and either the digitization template or the average shape of the sample (consensus) (Gunz et al., 2005; Gunz \& Mitteroecker, 2013). Finally, because asymmetry was not the 
subject of this study, data were symmetrized using reflected relabeling (Klingenberg, Barluenga, \& Meyer, 2002; Mardia, Bookstein, \& Moreton, 2000).

In order to evaluate the consistency of the digitization method and template, a random model was digitized three different times. Subsequently, we calculated the Procrustes distances between the three repetitions and compared them with the Procrustes distances of the whole sample. It is expected for the smallest Procrustes distance between two different configurations to be higher than any Procrustes distances between two different repetitions.

\section{3 | 3D geometric morphometric analyses}

Procrustes superimposition was carried out to center, scale, and rotate each configuration in order to remove all the morphological variation not due to shape (Gower, 1975). This process yielded Procrustes shape coordinates (Mitteroecker \& Gunz, 2009), which were statistically analyzed.

Differences in lumbar spine size were assessed by the centroid size-the square root of the sum of the squared distances between each landmark and their centroid (average of all coordinates) (Mitteroecker, Gunz, Windhager, \& Schaefer, 2013). We then calculated the mean centroid size for each of the sex and population groups. Normal distribution was assessed using Shapiro-Wilk test, and as not all subgroups were normally distributed (please, see results), statistical significance of size differences was tested by a Mann-Whitney $U$ test (Sokal \& Rohlf, 1998)-with a significance level of 0.05, using SPSS Statistics 21 software (http://www-01.ibm.com/ software/es/analytics/spss/).

Sexual shape dimorphism and inter-population shape variation of the lumbar vertebrae were both explored by carrying out a principal components analysis (PCA) in shape space (Mitteroecker et al., 2013; Zelditch et al., 2012). To determine statistically whether sex or interpopulation factors are associated with the shape variation of the lumbar spine explained by each principal component (PC), two dummy multivariate regressions of PC1 and PC2 shape variation were calculated (dummy variables: sex or population; 1,000 permutations, significance level 0.05) (Bastir et al., 2017; García-Martínez et al., 2018; Monteiro, 1999; Rosas \& Bastir, 2002; Torres-Tamayo et al., 2017) using MorphoJ software (Klingenberg, 2011). Dummy (or indicator) variables are created to represent an attribute which may have an impact on the sample's variability (i.e., sex or population) in a binary form (e.g., males $=1$; females $=-1$ ) in order to identify which factor influences the shape variation summarized in each PC. Once this was established, further Mann-Whitney tests were carried out to quantify the statistical differences between group shape means (sex or interpopulation factors) within PC1 and PC2 scores.

For lumbar spine shape visualization along each principal component $(\mathrm{PC})$ and comparison of the mean shape with the minimum and maximum ranges of each $\mathrm{PC}$ scores, we used the thin plate spline method (TPS) (Gunz \& Mitteroecker, 2013; Mitteroecker \& Gunz, 2009) in the EVAN Toolbox 1.63 software (http://www.evan-society. org/).

\section{4 | Cobb angle measurements}

We used the Cobb method (Cobb, 1948)-a standard method for quantification of the spinal curvature (Vrtovec et al., 2009)-to assess the lordotic curvature in our sample. Using the software Mimics 8 we produced reconstructed digital $2 \mathrm{D}$ images of the lumbar spines in the mid-sagittal plane from each of the subject's CT scans. We then applied the same technique as from a conventional radiograph to measure the lumbar Cobb angle (Brink et al., 2017). We drew two lines-one parallel to the superior endplate of L1, and another one parallel to the surface of the sacral endplate (S1) and measured the angle between them. In order to evaluate the reliability of our measurements, the Cobb angle was measured three different times by the same researcher, and the intraclass correlation coefficient (ICC) among the three different measurements was calculated (Langensiepen, Semler, \& Sobottke, 2013; Srinivasalu et al., 2008).

\section{3 | RESULTS}

\section{1 | Measurement error assessment}

The evaluation of the consistency of the digitization method shows that the smallest Procrustes distance between two different configurations (0.0282) was more than two times the maximum distance between two different repetitions (0.0137). Based on these results, we deemed the digitization method and template valid and of good quality for the identification and quantification of each individual model in the shape space of our sample.

\section{2 | Size analysis}

Normality tests of centroid size revealed normal distribution for the male and female sub-samples and also for the South African population sample but not for the Mediterranean population sample $(\mathrm{W}=0.88 ; p=.04)$. Mean centroid size of the lumbar spine was not statistically significantly different between the sexes (males: 1124.21, $S D=84.6$; females: $1089.95, S D=69.13$ ) (Mann-Whitney between sexes: $U=89, p=.242$ ). Furthermore, there is also no statistically significant mean centroid size difference between the Mediterranean $(1,121.7, S D=69.05)$ and South African $(1,088.07$, $S D=83.77$ ) population samples (Mann-Whitney between populations: $U=95, p=.333$ )

\section{3 | Shape analysis}

PCs 1 and 2 together summarize more than $60 \%$ of the total shape variability. PC1 (43.61\% of total variance) reflects variation in lordotic curvature: positive PC1 values correspond to less lordotic lumbar spines, while negative PC1 values are associated with greater lordotic curvatures (Figure 3; Movie S1; Movie S2). We observe that the less lordotic spines are a product of the combination of ventral wedging of L2 to L4 vertebral bodies and a more forwardly shifted position of L5, particularly its lower portion, as well as a somewhat greater dorsal 
(a)
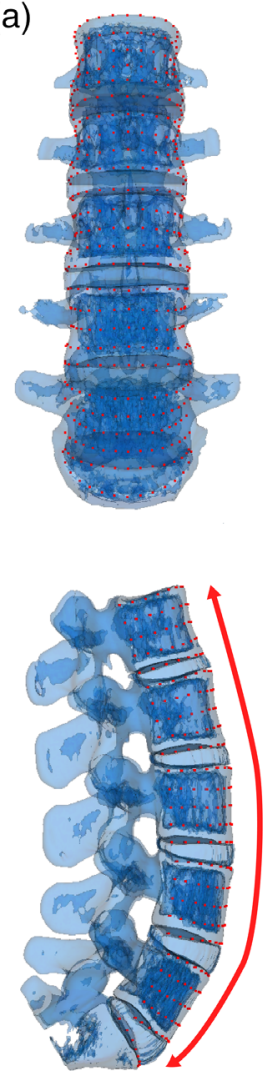

(b)
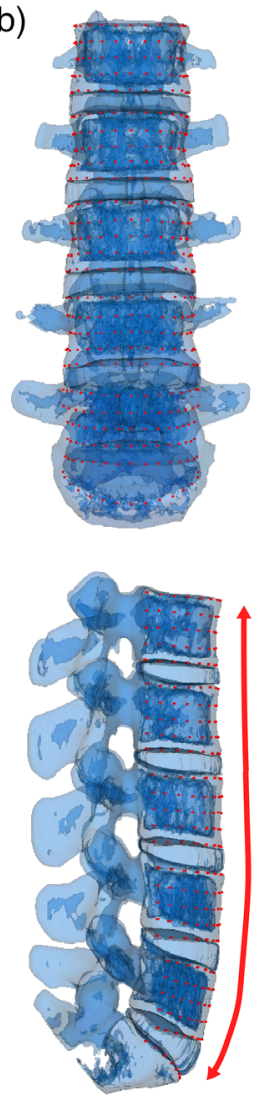

FIGURE 3 Shape differences explained by PC1, in frontal (top) and right lateral (bottom) views. (a) Negative values; (b) positive values. Arrows show the outline of the curvature and the differences in lordosis

wedging of the intervertebral disc L5-S1. It should be noted that the intervertebral discs of less lordotic spines are slightly taller (referring to craniocaudal height) and that the angle between the anterior surfaces of the $L 5$ body and the first sacral element is relatively smaller as well. In turn, a greater lordosis is produced by dorsal wedging of particularly L2 to L4 lumbar vertebrae, a retracted position of the L5 body, slightly relatively craniocaudally shorter intervertebral discs and dorsal wedging of especially disc L4-L5.

PC2 accounts for $23.91 \%$ of the total shape variance and summarizes variation in vertebral shape and shape proportions of vertebral bodies and intervertebral discs (Figure 4; Movie S3; Movie S4). It should be noted that all shape variation described and reported here is relative to the overall size of the spines rather than absolute. Negative PC2 scores are linked to craniocaudally relatively elongated vertebrae, where their transversal and dorsoventral lengths are relatively smaller than their vertical heights. Furthermore, the intervertebral spaces are relatively taller, thus, the discs seem to follow the same tendency as the vertebral bodies. Overall, it seems that intervertebral disc height scales positively with vertebral body height, particularly between L2-L4. Positive PC2 scores, on the other hand, are linked to relatively wider vertebrae, with relatively greater transversal and dorsoventral lengths and relatively shorter craniocaudal heights,

(a)
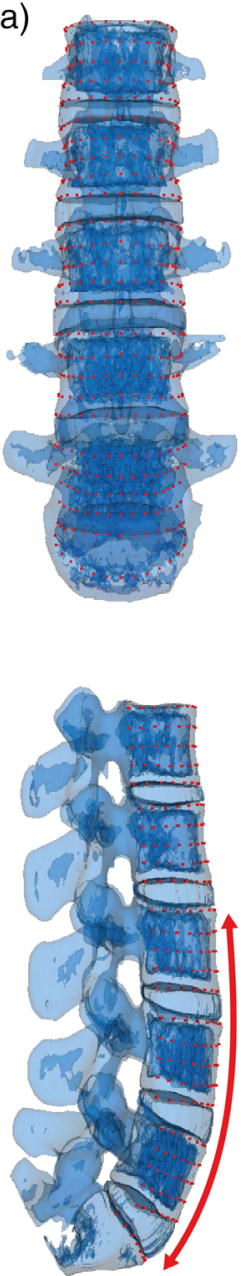

(b)
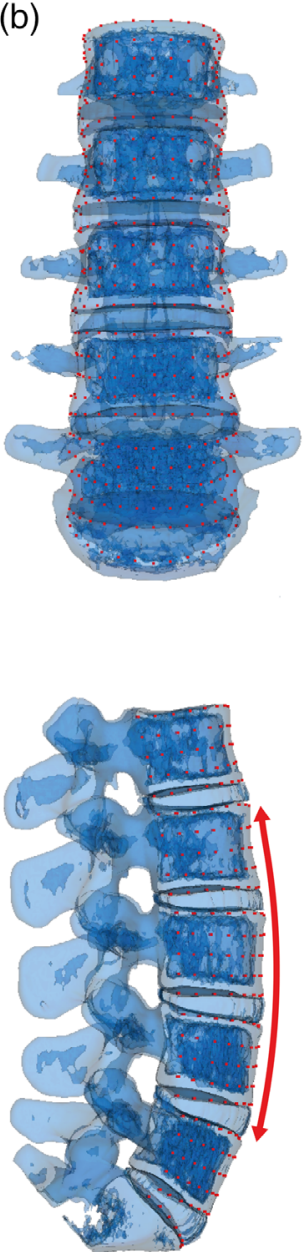

FIGURE 4 Shape differences explained by PC2, in frontal (top) and right lateral (bottom) views. (a) Negative values; (b) positive values. Arrows show the outline of the curvature and the position of the curvature maximum: Around L4-L5 for negative values and around L2-L3 for positive values

combined with relatively shorter intervertebral spaces, accommodating thinner intervertebral discs.

Interestingly, PC2 scores seem to be linked to slight variations in the spatial position of the lordosis (in the lateral view): negative PC scores indicate that the point of maximum curvature of the lumbar lordosis is located more caudally (at L4-L5), while positive PC scores point to a more cranially located curvature maximum close to L2-L3.

When projecting the Procrustes-aligned specimens in a tangent space along their principal component axes (PC1-PC2) (Figure 5), two different distributions of the sample along each axis are observed. The horizontal axis (PC1) shows a distribution related to differences between populations, where most of the Mediterraneans and their average shape plot toward negative values, and South Africans (and their average shape) plot toward positive ones. Lumbar lordosis variation contrasted by PC1 (Figure 5) suggests that the lumbar spines of Mediterraneans are more lordotic than those of South Africans. At the same time, the vertical axis (PC2) shows shape variation associated with sexual dimorphism. Males plot along positive values, which 
FIGURE 5 PC1-PC2 scatter plot. Larger symbols represent group means. Light blue: Mediterranean males; Light green: Mediterranean females; Dark blue: South African males; Dark green:

South African females

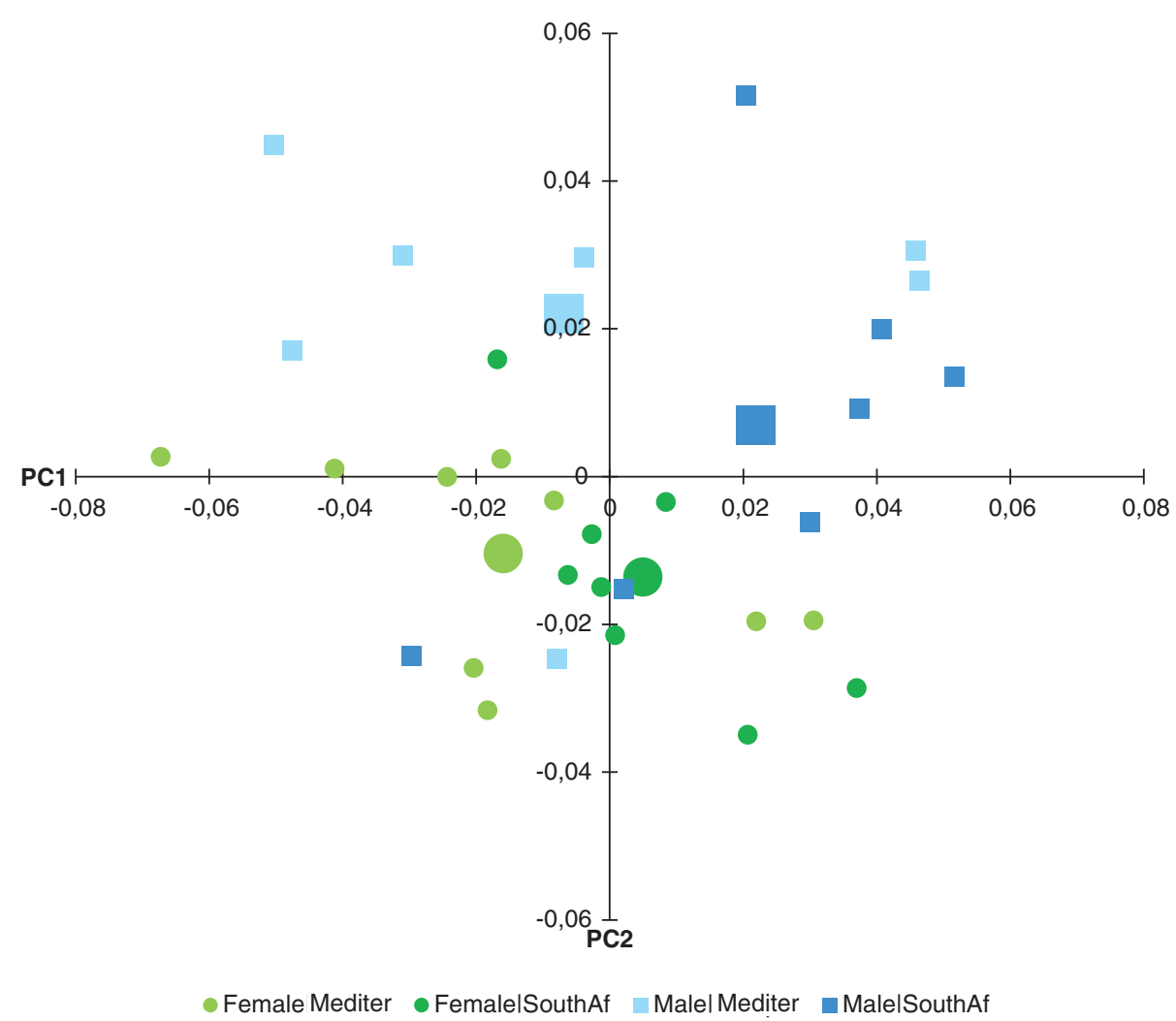

correspond to relatively wider vertebral bodies, while females are distributed along negative ones, corresponding to craniocaudally relatively elongated vertebrae.

The regression analyses of 3D coordinates on dummy variables statistically confirmed these associations. The inter-population factor accounts for $16.18 \%$ of total variance along PC1 scores ( $p=.033$ ), while the sex factor accounts for $32.93 \%$ of total shape variation along PC2 scores $(p=.003)$. The Mann-Whitney U tests between PC scores' means are also in accordance with these results: differences on PC1 scores between Mediterraneans and South Africans were statistically significant $(U=61 ; p=.020)$. The same is observed for differences on PC2 scores between males and females ( $U=45 ; p=.003)$.

\subsection{Cobb angle measurements}

The ICC used to assess measurement accuracy for the Cobb angle measurements was 0.99 , which reflects an excellent reliability (Langensiepen et al., 2013). Mean lordosis angles of each group measured by the Cobb method are indicated in Table 2 . The only statistically significant difference is observed between the different populations, where South Africans have smaller angles than Mediterraneans $(U=65 ; p=.03)$.

\section{4 | DISCUSSION}

This study investigated the 3D morphological variation of the adult lumbar spines from two different populations (Mediterraneans and
TAB LE 2 Mean lordosis angles of each group (Cobb method) and Mann-Whitney $U$ tests for differences between them

\begin{tabular}{|c|c|c|c|c|}
\hline & & & Mann-Whitney & \\
\hline & MEAN $\left({ }^{\circ}\right)$ & SD & $\mathbf{U}$ & $p$-value \\
\hline Mediterranean males & 49.47 & 14.25 & \multirow[t]{2}{*}{27} & \multirow[t]{2}{*}{.634} \\
\hline Mediterranean females & 55.21 & 9.95 & & \\
\hline South African males & 43.05 & 11.33 & \multirow[t]{2}{*}{23} & \multirow[t]{2}{*}{.563} \\
\hline South African females & 45.48 & 7.5 & & \\
\hline Total males & 46.26 & 12.81 & \multirow[t]{2}{*}{97} & \multirow[t]{2}{*}{.383} \\
\hline Total females & 50.63 & 9.96 & & \\
\hline Total Mediterranean & 52.7 & 11.95 & \multirow[t]{2}{*}{65} & \multirow[t]{2}{*}{$.03 *$} \\
\hline Total South African & 44.35 & 9.2 & & \\
\hline TOTAL & 48.66 & 11.17 & - & - \\
\hline
\end{tabular}

Note: Statistically significant values $(p<.05)$ are marked with an asterisk (*).

South Africans). The purpose was to evaluate the morphology and curvature of the lumbar spine (vertebral bodies and discs) through 3D geometric morphometrics, exploring two potential causes of variation such as shape variability due to different genetic backgrounds and sexual dimorphism, and to compare our results with the Cobb angle method.

\subsection{Geometric versus traditional morphometrics}

Our results on classic measurements (Table 2) are consistent with the 3D-GM findings: while in the population analysis angular differences were statistically significant, in the sexual dimorphism analysis they 
were not. It should be noted that the total sample size $(N=31)$ used here is smaller than in other studies where the Cobb's method is applied (Been et al., 2010; Kalichman et al., 2011; Shefi et al., 2013; Vialle et al., 2005), which likely influences the results of the statistical tests.

When compared with classical measurements, we would like to emphasize that 3D-GM analysis does not only show differences in curvature degree but also other features, for example, anterior and posterior heights of the vertebral bodies. This is important because a given lordotic angle only describes the angular relation between L1 and S1 superior endplates, while the shape of the full lumbar curvature (quantified by PC1) between these endplates can be variable in other different aspects. For example, different vertebral body heights (in different orientations) could cause different curvature outlines within the same angle. Alternatively, differences in vertebral wedging and the inclination of bodies at different lumbar levels could modify lumbar curvature shape but not necessarily its angle. Furthermore, we should not forget that these spatial arrangements are closely related and even dependent from the sacrum and pelvis position within the trunk (Been et al., 2017; Vaz et al., 2002; Vialle et al., 2005).

\subsection{Sexual dimorphism in the lumbar spine}

The largest lumbar spine shape variation we found is associated with the lordotic curvature-as shown by PC1. This is to be expected, as the main direction of mobility and the widest range of movement of the lumbar spine occurs in the sagittal plane and is achieved by variation of the lordosis. As mentioned before, several authors reported statistically significant sexual dimorphism in this curvature (Bailey et al., 2016; Poussa et al., 2005; Vialle et al., 2005; Whitcome et al., 2007). However, together with other authors who did not find any statistically meaningful differences between the degrees of male and female lordosis (Fazzalari et al., 2001; Kalichman et al., 2011) we did not observe statistically significant difference in the degree of the lordosis angle.

More importantly, our results show that sexual dimorphism is reflected more powerfully by morphological factors other than variation in lordosis angle degree, as seen in PC2 which shows sex-specific variation that is similar in both populations (Figure 4; Movie S3; Movie S4). Significant sexual dimorphism is expressed here by vertebral body morphology and proportions. Female vertebrae appear to be relatively elongated craniocaudally with respect to their horizontal measures, while male vertebrae show relatively greater transversal and dorsoventral lengths in relation to vertebral height (Figure 4). These different proportions were already observed by Taylor and Twomey (1984) who reported a smaller height/transverse diameter index in males. Wider vertebrae lead to a more robust appearance of the male spine, concordant with the larger male skeleton, necessary to support and move larger body sizes and weights and which is developed via larger growth rate and/or a longer growth period than what is observed in females (Bastir, Higuero, Ríos, \& García-Martínez, 2014; Bogin, 1999; Bulygina, Mitteroecker, \& Aiello, 2006). These different height-width ratios seem to reflect sexual dimorphism of the entire vertebral column, as such pattern of morphological differences is also found in the thoracic vertebrae (Bastir et al., 2014).

Furthermore, Figure 4 (see also Movie S4) also indicates very slight variation in lordosis morphology in a spatial sense: in males, the point of maximum lordotic curvature is located more cranially (L2-L3) within the lumbar spine than in females (L4-L5). Therefore, the overall shape outline of the lumbar spine is different between the sexes. A similar trend has been observed in the cervical region of the spine as well. Been et al. (2017) compared the cervical lordosis of young and adult individuals of both sexes and found that while total cervical lordosis was similar in males and females, males showed smaller upper cervical lordosis and higher lower cervical lordosis, whereas females showed the opposite outline. This would suggest some overarching integrative pattern of spine curvatures and might be linked to the sexual dimorphism observed in the onset of the adolescent growth spurt between male and females (e.g., Schlösser, Vincken, Rogers, Castelein, \& Shah, 2015; Shefi et al., 2013; Voutsinas \& Macewen, 1986; Willner \& Johnson, 1983). Further, detailed investigations of the thoracic region and the vertebral column as a whole would help clarify this.

In conclusion, the main features affected by sexual dimorphism as suggested by our results are vertebral body robustness and overall lumbar spine shape outline.

\section{3 | Population specific features of lumbar spine variation}

In our sample, the inter-population variability seems to have a stronger effect on the lumbar curvature than sexual dimorphism, as it is represented on the first principal component. This is observed in the PCA results (Figure 5), as well as in the classic measurements (Table 2) and complements other studies. As mentioned in the introduction, there are few and variable studies that address the influence of inter-population variability on the morphology of the lumbar spine in several aspects. Mosner et al. (1989) and Merrill et al. (2018) did not find differences between the actual lordosis of an African-American and a European-American population. Neither did Le Huec and Hasegawa (2016) between a Caucasian and a Japanese population. Patrick (1976) compared a Nigerian population with a European one, and found a greater lumbar curvature in the former. Okpala (2014) found that his Nigerian population had a statistically significantly larger sacral slope when compared to several other studies based on non-Nigerian samples and investigated with a similar methodology (while not always in the same supine position). While Patricks' and Okpala's comparison of a Nigerian and a European population is not the same as comparing our Mediterranean population with the South African sample, our results nonetheless confirm that the inter-population variability is an important factor when studying lumbar spine morphology.

We should point out that, besides genetic factors, these differences in study outcomes might be also attributed to the very different data and methods used by each author as suggested also by Hanson et al. 
(1998). Mosner et al. (1989) investigated the difference between evaluating the lumbar curvature by studying the body contour (apparent lordosis) and evaluating this curvature by actually studying the lumbar spine through a lateral radiograph (actual lordosis), where they calculated the angle between a line parallel to the superior surface of the L2 and another one either parallel to the inferior surface of the L5 (lumbolumbar angle) or the sacral surface (lumbosacral angle). Merrill et al. (2018) also used lateral radiographs but calculated the angle between the L1 vertebra and the sacral surface (L1-S1 lordosis). Le Huec and Hasegawa (2016) performed a study based on EOS images. Patrick (1976) used a flexicurve ruler (Milne \& Lauder, 1974) to measure the curvature on the back of standing subjects, from the seventh cervical vertebra to the lumbosacral joint, both located by palpation on external body surface. Okpala (2014) used radiographs to measure the lumbosacral angle of each lumbar spine, formed between a line parallel to the S1 endplate and a horizontal line.

Our 3G-GM study enabled us to demonstrate that different morphological variations of the lumbar curvature are reflected both in PC1 (stronger vs. weaker curvature degree) and PC2 (more cranially located vs. more caudally located point of maximum curvature). The 3D morphometrics approach therefore enables us to differentiate between different structural patterns contributing to the formation of the lordosis. Lordosis as linked to the inter-population factor (PC1) lacks the associated component of spatial position and variation in robustness, whereas lordosis related to sexual dimorphism (PC2), is linked with differences in the position of the curvature maximum.

The fact that our Mediterranean sample is more lordotic than the South African sample, or rather, that the variability in the adult lordotic curvature could be affected by other factors such as inter-population variation, may hence suggest that explaining sexual dimorphism of lordosis only (or mainly) in terms of adaptation to pregnancy is insufficient. Clearly, other factors such as the spino-pelvic alignment, that is, the morphology of the pelvis, the pelvic incidence, the sacral slope and the relation of the sacrum relative to the lumbar spine (Been et al., 2017; Vaz et al., 2002; Vialle et al., 2005), body size, body mass index and body shape all have an impact.

Beside these factors, and others related to the variability of the lumbar spine as subject of study-number of vertebrae used, pathological or nonpathological spines, presence or absence of lumbosacral transitional vertebrae, presence or absence of scoliosis, and so forth, discrepancies found among studies of sexual dimorphism and population variation could also stem from differences in the inclusion/exclusion criteria used by each author, which are often based on the sample's age range. Some consider age-related degeneration to have an influence on the lumbar curvature-either increasing it (Jentzsch et al., 2017) or decreasing it (Dreischarf et al., 2014; Gelb, Lenke, Bridwell, Blanke, \& McEnery, 1995; Korovessis et al., 1998; Merrill et al., 2018; Pries et al., 2015)-and prefer to choose a younger-aged sample in order to eliminate its effect in studies where age variation is not the main subject of study (Bailey et al., 2016). On the contrary, other authors do not find any relation between lordosis and age (Kalichman et al., 2011), and thus do not make any exclusions regarding age. However, sexually dimorphic variation in postnatal ontogenetic trajectories and growth patterns will need to be considered as well, as these variations have been reported but need to be further assessed in relation to the lumbar vertebral morphology (Schlösser et al., 2015; Shefi et al., 2013; Voutsinas \& Macewen, 1986).

\section{4 | Study limitations}

The influence of posture on the degree of the spinal curvature parameters needs to be monitored closely: It has been reported that the lordotic angle tends to be larger in a standing position than in a supine position (Chevillotte et al., 2018; Mauch, Jung, Huth, \& Bauer, 2010). This is most likely because axial loads (e.g., gravity forces, body weight) act on the soft tissue-intervertebral discs mostly-and flatten the lumbar curvature in the supine position (Bailey et al., 2016). Therefore, posture could be another cause of discrepancy found in the results between various studies, and should be taken into account. We would caution that our results could only be compared with those from studies based on the same approach to data collection (i.e., data collected from subjects measured in the supine position).

A further limitation of this study is its small sample size $(N=31)$ which likely influences the results of the statistical tests taken both for classical measures and 3D-GM analysis, and so the study could benefit from a larger sample.

\section{5 | Future steps}

This study investigated the lumbar vertebral bodies and the intervertebral discs and therefore the part of the lumbar vertebral column most associated with weight transfer and stability. In a further step, the posterior aspect of the lumbar vertebral column, associated with spinal region mobility and shape variation (e.g., degree of lordosis) could be studied as well. This would include the vertebral processes (costal, spinous, and articular processes) and intervertebral joint morphology and would further advance our understanding of how these vertebral elements integrate and interact with regards to variation in the $3 \mathrm{D}$ morphology of the human axial skeleton.

\section{ACKNOWLEDGMENTS}

We would like to thank the Paleoanthropology group of the National Museum of Natural History (MNCN-CSIC) for their support, and the radiology services from Mount Carmel Hospital in Haifa, ERESA Medical Group for Special Radiological Explorations and Charlotte Maxeke Academic Hospital for the providing of the CT images; also to Dr. Benoît Beyer for his feedback and contributions. This research is funded by CGL-2015-63648-P (Ministry of Economy, Industry and Competitiveness, Spain) and Project BF14_005 (ERESA Clinic Research Foundation Valencia). We also thank the anonymous reviewers for their helpful comments on previous drafts. 


\section{AUTHOR'S CONTRIBUTIONS}

Designed the project: M.B. Performed the analyses: S.L.Z. Analyzed the results: M.B. and S.L.Z. Contributed materials/analyses: N.T.T., D.G.M., E.B.P., F.M., A.B, S.N., and J.A.S.G. Wrote the manuscript: S.L.Z., M.B., and S.M. Critical revision of the article/discussion: S.L.Z, M.B., S.M., N.T.T., D.G.M., J.A.S.G., and S.N.

\section{ORCID}

Stephanie Lois Zlolniski (ID https://orcid.org/0000-0003-2721-5848 Nicole Torres-Tamayo (D) https://orcid.org/0000-0003-2504-3886 Daniel García-Martínez (D) https://orcid.org/0000-0001-7518-3866 Markus Bastir (D) https://orcid.org/0000-0002-3141-3401

\section{REFERENCES}

Bailey, J. F., Sparrey, C. J., Been, E., \& Kramer, P. A. (2016). Morphological and postural sexual dimorphism of the lumbar spine facilitates greater lordosis in females. Journal of Anatomy, 229(1), 82-91.

Bastir, M., García-Martínez, D., Torres-Tamayo, N., Sanchis-Gimeno, J. A., O'Higgins, P., Utrilla, C., ... García-Río, F. (2017). In vivo 3D analysis of thoracic kinematics: Changes in size and shape during breathing and their implications for respiratory function in recent humans and fossil hominins. The Anatomical Record, 300, 255-264. https://doi.org/10. 1002/ar.23503

Bastir, M., Higuero, A., Ríos, L., \& García-Martínez, D. (2014). Threedimensional analysis of sexual dimorphism in human thoracic vertebrae: Implications for the respiratory system and spine morphology. American Journal of Physical Anthropology, 155, 513-521.

Been, E., Barash, A., \& Marom, A. (2010). Vertebral bodies or discs. Which contributes more to human-like lumbar lordosis? Clinical Orthopaedics and Related Research, 468, 1822-1829.

Been, E., Gómez-Olivencia, A., Shefi, S., Soudack, M., Bastir, M., \& Barash, A. (2017). Evolution of spinopelvic slignment in hominins. The Anatomical Record, 300(5), 900-911.

Behar, D. M., Rosset, S., Blue-Smith, J., Balanovsky, O., Tzur, S., Comas, D., ... Wells, R. S. (2007). The Genographic project public participation mitochondrial DNA database. PLoS Genetics, 3(9), e169. https://doi. org/10.1371/journal.pgen.0030169

Bittersohl, B., Freitas, J., Zaps, D., Schmitz, M. R., Bomar, J. D., Muhamad, A. R., \& Hosalkar, H. S. (2013). EOS imaging of the human pelvis: Reliability, validity, and controlled comparison with radiography. The Journal of Bone \& Joint Surgery, 95(9), e58. https://doi.org/10. 2106/JBJS.K.01591

Bogduk, N. (2005). Clinical anatomy of the lumbar spine and the sacrum. Edinburgh: Elsevier.

Bogin, B. (1999). Patterns of human growth. Cambridge: Cambridge University Press.

Bookstein, F. L. (1991). Morphometric tools for landmark data: Geometry and biology. New York: Cambridge University Press.

Brink, R. C., Colo, D., Schlösser, T. P. C., Vincken, K. L., Stralen, M., Hui, S. C. N., ... Castelein, R. M. (2017). Upright, prone, and supine spinal morphology and alignment in adolescent idiopathic scoliosis. Scoliosis and Spinal Disorders, 12, 6. https://doi.org/10.1186/s13013-0170111-5

Bulygina, E., Mitteroecker, P., \& Aiello, L. (2006). Ontogeny of facial dimorphism and patterns of individual development within one human population. American Journal of Physical Anthropology, 144, 238-247.

Chevillotte, T., Coudert, P., Cawley, D., Bouloussa, H., Mazas, S., Boissière, L., \& Gille, O. (2018). Influence of posture on relationships between pelvic parameters and lumbar lordosis: Comparison of the standing, seated, and supine positions. A preliminary study. Orthopaedics \& Traumatology: Surgery \& Research, 104(5), 565-568. https://doi. org/10.1016/j.otsr.2018.06.005

Chiron, P., Demoulin, L., Wytrykowski, K., Cavaignac, E., Reina, N., \& Murgier, J. (2017). Radiation dose and magnification in pelvic X-ray: EOS $^{\mathrm{TM}}$ imaging system versus plain radiographs. Orthopaedics \& Traumatology: Surgery \& Research, 103(8), 1155-1159. https://doi.org/ 10.1016/j.otsr.2017.07.018

Cobb, J. (1948). Outline for the study of scoliosis. American Academy of Orthopaedic Surgeons Instructional Course Lectures, 5, 261-275.

Dreischarf, M., Albiol, L., Rohlmann, A., Pries, E., Bashkuev, M., Zander, T., ... Schmidt, H. (2014). Age-related loss of lumbar spinal lordosis and mobility - A study of 323 asymptomatic volunteers. PLoS One, 9(12), e116186. https://doi.org/10.1371/journal.pone.0116186

Dubousset, J., Charpak, G., Dorion, I., Skalli, W., Lavaste, F., Deguise, J., ... Ferey, S. (2005). Une nouvelle imagerie ostéo-articulaire basse dose en position debout: Le système EOS. Radioprotection, 40(2), 245-255. https://doi.org/10.1051/radiopro:2005010

Farfan, H. F. (1995). Form and function of the musculoskeletal system as revealed by mathematical analysis of the lumbar spine. An essay. Spine, 20, 1462-1474.

Fazzalari, N., Manthey, B., \& Parkinson, I. (2001). Intervertebral disc disorganization and its relationship to age adjusted vertebral body morphometry and vertebral bone architecture. The Anatomical Record, 262, 331-339.

García-Martínez, D., Radovčić, D., Radovčić, J., Cofran, Z., Rosas, A., \& Bastir, M. (2018). Over 100 years of Krapina: New insights into the Neanderthal thorax from the study of rib cross-sectional morphology. Journal of Human Evolution, 122, 124-132.

Gelb, D. E., Lenke, L. G., Bridwell, K. H., Blanke, K., \& McEnery, K. W. (1995). An analysis of sagittal spinal alignment in 100 asymptomatic middle and older aged volunteers. Spine, 20(12), 1351-1358.

Goodyear, M., Krleza-Jeric, K., \& Lemmens, T. (2007). The declaration of Helsinki. BMJ, 335, 736.

Gower, J. C. (1975). Generalized procrustes analysis. Psychometrika, 40(1), 33-51.

Gunz, P., \& Mitteroecker, P. (2013). Semilandmarks: A method for quantifying curves and surfaces. Hystrix, the Italian Journal of Mammalogy, 24, 103-109.

Gunz, P., Mitteroecker, P., \& Bookstein, F. L. (2005). Semilandmarks in three dimensions. In D. E. Slice (Ed.), Modern morphometrics in physical anthropology (pp. 73-98). New York: Kluwer Academic/ Plenum Publishers.

Hanson, P., Magnusson, S. P., \& Simonsen, E. B. (1998). Differences in sacral angulation and lumbosacral curvature in black and white young men and women. Acta Anatomica, 162, 226-231.

Hasegawa, K., Okamoto, M., Hatsushikano, S., Shimoda, H., Ono, M. Homma, T., \& Watanabe, K. (2017). Standing sagittal alignment of the whole axial skeleton with reference to the gravity line in humans. Journal of Anatomy, 230, 619-630.

Hellems, H. K., \& Keats, T. E. (1971). Measurement of the normal lumbosacral angle. The American Journal of Roentgenology Radium Therapy and Nuclear Medicine, 113, 642-645.

Jentzsch, T., Geiger, J., König, M. A., \& Werner, C. M. L. (2017). Hyperlordosis is associated with facet joint pathology at the lower lumbar spine. Clinical Spine Surgery, 30, 129-135.

Kalichman, L., Li, L., Hunter, D. J., \& Been, E. (2011). Association between computed tomography-evaluated lumbar lordosis and features of spinal degeneration, evaluated in supine position. The Spine Journal, 11, 308-315.

Klingenberg, C. P. (2011). MorphoJ: An integrated software package for geometric morphometrics. Molecular Ecology Resources, 11, 353-357. 
Klingenberg, C. P., Barluenga, M., \& Meyer, A. (2002). Shape analysis of symmetric structures: Quantifying variation among individuals and asymmetry. Evolution, 56(10), 1909-1920.

Korovessis, P. G., Stamatakis, M. V., \& Baikousis, A. G. (1998). Reciprocal angulation of vertebral bodies in the sagittal plane in an asymptomatic Greek population. Spine, 23(6), 700-704 discussion 704-705.

Kummer, B. K. F. (1975). Functional adaptation to posture in the pelvis of man and other primates. In R. H. Tuttle (Ed.), Primate functional morphology and evolution (pp. 281-290). The Hague: Mouton.

Langensiepen, S., Semler, O., \& Sobottke, R. (2013). Measuring procedures to determine the Cobb angle in idiopathic scoliosis: A systematic review. European Spine Journal, 22, 2360-2371.

Le Huec, J. C., \& Hasegawa, K. (2016). Normative values for the spine shape parameters using 3D standing analysis from a database of 268 asymptomatic Caucasian and Japanese subjects. European Spine Journal, 25, 3630-3637.

Legaye, J., Duval-Beaupère, G., Hecquet, J., \& Marty, C. (1998). Pelvic incidence: A fundamental pelvic parameter for three-dimensional regulation of spinal sagittal curves. European Spine Journal, 7, 99-103.

Lois Zlolniski, S., García-Martínez, D., Blanco-Pérez, E., SanchisGimeno, J. A., Barash, A., Martelli, S., ... Bastir, M. (2017). 3D geometric morphometrics of lumbar vertebral curvatures in $\mathrm{H}$. sapiens. American Journal of Physical Anthropology, 162, 264-265.

Lovejoy, C. O. (2005). The natural history of human gait and posture: Part 1. Spine and pelvis. Gait \& Posture, 21(1), 95-112. https://doi.org/10. 1016/j.gaitpost.2004.01.001

Maduforo, C., West, O., Nwankwo, N., Onwuchekwa, R., Etawo, U. S., \& Ogbulu, D. (2012). Study of the lumbosacral angles of males in Port Harcourt, south-south, Nigeria. Nigerian Health Journal, 12, 22-24.

Mardia, K. V., Bookstein, F. L., \& Moreton, I. J. (2000). Statistical assessment of bilateral symmetry of shapes. Biometrika, 87, 285-300.

Masharawi, Y., Dar, G., Peleg, S., Steinberg, N., Medlej, B., May, H., ... Hershkovitz, I. (2010). A morphological adaptation of the thoracic and lumbar vertebrae to lumbar hyperlordosis in young and adult females. European Spine Journal, 19, 768-773.

Mauch, F., Jung, C., Huth, J., \& Bauer, G. (2010). Changes in the lumbar spine of athletes from supine to the true-standing position in magnetic resonance imaging. Spine, 35(9), 1002-1007. https://doi.org/10.1097/ BRS.0b013e3181bdb2d3

Merrill, R. K., Kim, J. S., Leven, D. M., Kim, J. H., Meaike, J. J., Bronheim, R. S., ... Cho, S. K. (2018). Differences in fundamental sagittal pelvic parameters based on age, sex and race. Clinical Spine Surgery, 31(2), E109-E114.

Milne, J. S., \& Lauder, I. J. (1974). Age effects in kyphosis and lordosis in adults. Annals of Human Biology, 1, 327-337. https://doi.org/10.1080/ 03014467400000351

Mitteroecker, P., \& Gunz, P. (2009). Advances in geometric morphometrics. Evolutionary Biology, 36, 235-247.

Mitteroecker, P., Gunz, P., Windhager, S., \& Schaefer, K. (2013). A brief review of shape, form, and allometry in geometric morphometrics, with applications to human facial morphology. Hystrix, the Italian Journal of Mammalogy, 24(1), 59-66.

Monteiro, L. R. (1999). Multivariate regression models and geometric morphometrics: The search for causal factors in the analysis of shape. Systematic Biology, 48(1), 192-199. https://doi.org/10.1080/106351599260526

Mosner, E. A., Bryan, J. M., Stull, M. A., \& Shippee, R. (1989). A comparison of actual and apparent lumbar lordosis in black and white adult females. Spine, 14, 310-314.

O'Higgins, P. (2000). The study of morphological variation in the hominid fossil record: Biology, landmarks and geometry. Journal of Anatomy, 197, 103-120.

Okpala, F. O. (2014). Measurement of lumbosacral angle in normal radiographs: A retrospective study in Southeast Nigeria. Annals of Medical and Health Sciences Research, 4, 757-762.
Patrick, J. M. (1976). Thoracic and lumbar spinal curvatures in Nigerian adults. Annals of Human Biology, 3, 383-386.

Poussa, M. S., Heliövaara, M. M., Seitsamo, J. T., Könönen, M. H., Hurmerinta, K. A., \& Nissinen, M. J. (2005). Development of spinal posture in a cohort of children from the age of 11 to 22 years. European Spine Journal, 14, 738-742.

Pries, E., Dreischarf, M., Bashkuev, M., Putzier, M., \& Schmidt, H. (2015). The effects of age and gender on the lumbopelvic rhythm in the sagittal plane in 309 subjects. Journal of Biomechanics, 48, 3080-3087.

Reichmann, S., \& Lewin, T. (1971). The development of the lumbar lordosis, a post mortem study on excised lumbar spines. Archiv für Orthopädische Und Unfall-Chirurgie, 69, 275-285.

Rosas, A., \& Bastir, M. (2002). Thin-plate spline analysis of allometry and sexual dimorphism in the human craniofacial complex. American Journal of Physical Anthropology, 117, 236-245.

Schlösser, T., Vincken, K., Rogers, K., Castelein, R., \& Shah, S. (2015). Natural sagittal spino-pelvic alignment in boys and girls before, at and after the adolescent growth spurt. European Spine Journal, 24(6), 1158-1167.

Shefi, S., Soudack, M., Konen, E., \& Been, E. (2013). Development of the lumbar lordotic curvature in children from age 2 to 20 years. Spine, 38 (10), E602-E608.

Sokal, R. R., \& Rohlf, F. J. (1998). Biometry (3rd ed.). New York: W. H. Freeman and Company.

Srinivasalu, S., Modi, H. N., SMehta, S., Suh, S.-W., Chen, T., \& Murun, T. (2008). Cobb angle measurement of scoliosis using computer measurement of digitally acquired radiographs-intraobserver and interobserver variability. Asian Spine Journal, 2(2), 90-93. https://doi.org/10.4184/ asj.2008.2.2.90

Tardieu, C., Hasegawa, K., \& Haeusler, M. (2017). How did the pelvis and vertebral column become a functional unit during the transition from occasional to permanent bipedalism? The Anatomical Record, 300, 912-931.

Taylor, J. R., \& Twomey, L. T. (1984). Sexual dimorphism in human vertebral body shape. Journal of Anatomy, 138(2), 281-286.

Torres-Tamayo, N., García-Martínez, D., Lois Zlolniski, S., TorresSánchez, I., García-Río, F., \& Bastir, M. (2017). 3D analysis of sexual dimorphism in size, shape and breathing kinematics of human lungs. Journal of Anatomy, 232(2), 227-237. https://doi.org/10.1111/joa. 12743

Torres-Tamayo, N., García-Martínez, D., Nalla, S., Barash, A., Williams, S. A., Blanco-Pérez, E., ... Bastir, M. (2018). The torso integration hypothesis revisited in Homo sapiens: Contributions to the understanding of hominin body shape evolution. American Journal of Physical Anthropology, 167(4), 777-790. https://doi.org/10.1002/ ajpa.23705

Turner, W. (1886). The human curve of the spinal column in several races of men. Journal of Anatomy and Physiology, 20, 536-543.

Vaz, G., Roussouly, P., Berthonnaud, E., \& Dimnet, J. (2002). Sagittal morphology and equilibrium of pelvis and spine. European Spine Journal, 11 (1), 80-87. https://doi.org/10.1007/s005860000224

Vialle, R., Levassor, N., Rillardon, L., Templier, A., Skalli, W., \& Guigui, P. (2005). Radiographic analysis of the sagittal alignment and balance of the spine in asymptomatic subjects. The Journal of bone and joint surgery. American Volume, 87(2), 260-267.

Voutsinas, S., \& Macewen, G. (1986). Sagittal profiles of the spine. Clinical Orthopaedics and Related Research, 210, 235-242.

Vrtovec, T., Pernus, F., \& Likar, B. (2009). A review of methods for quantitative evaluation of spinal curvature. European Spine Journal, 18, 593-607.

Whitcome, K. K., Shapiro, L. J., \& Lieberman, D. E. (2007). Fetal load and the evolution of lumbar lordosis in bipedal hominins. Nature, 450, 1075-1078. 
Willner, S. (1981). Spinal pantograph-a non-invasive technique for describing kyphosis and lordosis in the thoraco-lumbar spine. Acta Orthopaedica Scandinavica, 52(5), 525-529. https://doi.org/10.3109/ 17453678108992142

Willner, S., \& Johnson, B. (1983). Thoracic kyphosis and lumbar lordosis during the growth period in children. Acta Paediatrica Scandinavica, 72, 873-878.

Zelditch, M. L., Swiderski, D. L., \& Sheets, H. D. (2012). Geometric Morphometrics for biologists: A primer. San Diego, EEUU: Elsevier.

Zhou, S. H., McCarthy, I. D., McGregor, A. H., Coombs, R. R. H., \& Hughes, S. P. F. (2000). Geometrical dimensions of the lower lumbar vertebrae - Analysis of the data from digitised CT images. European Spine Journal, 9, 242-248.

\section{SUPPORTING INFORMATION}

Additional supporting information may be found online in the Supporting Information section at the end of this article.

How to cite this article: Lois Zlolniski S, Torres-Tamayo N, García-Martínez D, et al. 3D geometric morphometric analysis of variation in the human lumbar spine. Am J Phys Anthropol. 2019;170:361-372. https://doi.org/10.1002/ajpa.23918 